Images du travail, travail des images

1 | 2016

Quand les groupes professionnels se mettent en images

\title{
Les façades symboliques du conseil en gestion. Mise en image et mise en récit d'un groupe professionnel
}

Isabel Boni-Le Goff

\section{OpenEdition}

Journals

Édition électronique

URL : http://journals.openedition.org/itti/1325

DOI : $10.4000 /$ itti. 1325

Éditeur

Université de Poitiers

Référence électronique

Isabel Boni-Le Goff, "Les façades symboliques du conseil en gestion. Mise en image et mise en récit d'un groupe professionnel », Images du travail, travail des images [En ligne], 1 | 2016, mis en ligne le 01 février 2016, consulté le 14 avril 2021. URL : http://journals.openedition.org/itti/1325 ; DOI : https:// doi.org/10.4000/itti.1325

Ce document a été généré automatiquement le 14 avril 2021.

Images du travail, travail des images 


\title{
Les façades symboliques du conseil en gestion. Mise en image et mise en récit d'un groupe professionnel
}

\author{
Isabel Boni-Le Goff
}

1 Le management est souvent analysé comme un ensemble de «techniques de gouvernement» (Foucault, 2001) dont la capacité à se propager à l'ensemble des sphères sociales sur la période contemporaine, a fait l'objet d'un corpus conséquent de travaux (Chandler, 1988 ; Boussard, 2008). Mais ces techniques de gouvernement sont souvent présentées comme des techniques discursives, des " outils », des «méthodes » et ceux qui en assurent la diffusion, en particulier les "spécialistes du management » que disent être les consultants en management, semblent parfois désincarnés (Kerfoot et Knights, 1996).

2 La rhétorique de ces professionnels insiste d'ailleurs surtout sur les dimensions intellectuelles et immatérielles du travail réalisé. L'observation des pratiques professionnelles livre pourtant un tout autre constat et souligne la place occupée par le travail relationnel en coprésence, la « co-production» (Gadrey 2003), et les formes de dramaturgie entourant la mise en jeu et la circulation de l'expertise gestionnaire. C'est cet apparent paradoxe qu'une recherche doctorale effectuée sur le conseil en management et sur les divisions sexuées au sein de cet espace, a notamment exploré, mettant en lumière la place importante occupée par l'image, dans la communication institutionnelle et personnelle réalisée par les professionnel-le-s du conseil en management. Avec les photographies mises en ligne sur les sites internet des firmes de conseil ou sur les réseaux sociaux à usage professionnel, c'est à une véritable mise en scène collective des apparences a laquelle on assiste quand on s'attarde sur les sites des cabinets, quels que soient leur taille ou leur prestige.

Production éminemment sociale, avec ses destinataires et ses objectifs, (Terrenoire 1985 ; Géhin et Stevens, 2012), les images du travail donnent souvent plus à voir les intentions et l'orientation du regard, que le réel du travail. C'est bien en resituant ces images comme des construits sociaux, que le présent article souhaite articuler deux 
grands questionnements: Dans un premier temps, quel(s) récit(s) les images construisent-elles du métier de conseil en gestion? En quoi ces récits peuvent-ils être une ressource pour légitimer une expertise faiblement institutionnalisée et symboliser un travail en partie invisible ? Puis dans second temps que produisent ces images pour les professionnel-le-s ? Quelles normes d'apparence, d'hexis corporelle et vestimentaire construisent-elles? Et quels en sont symboliquement et pratiquement les effets, notamment en termes de construction de figures professionnelles « corporellement » légitimes et de genre?

Photographie 1. CS2 and Partners

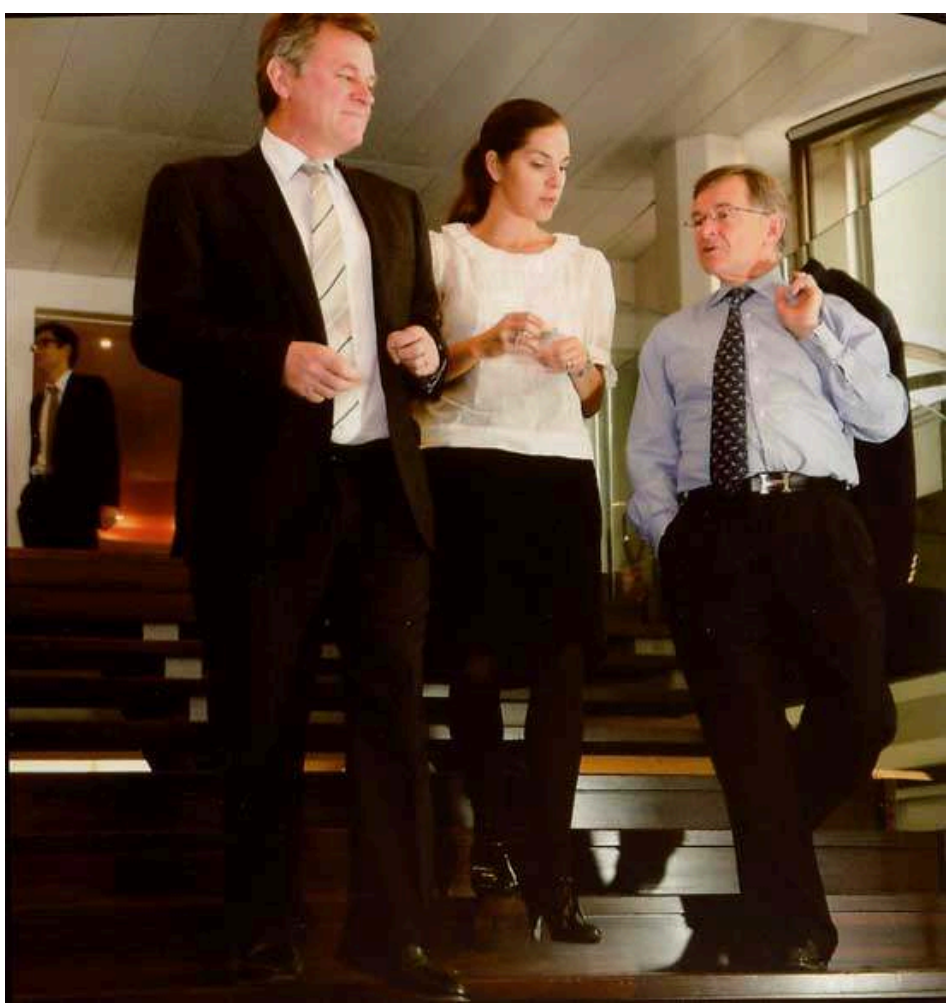

En s'appuyant sur un matériau d'enquête composé de deux groupes de ressources iconographiques (voir encadré 1), on montrera d'abord combien l'image revêt dès l'émergence de ce groupe professionnel et jusqu'à aujourd'hui, des enjeux importants, et participe à sa construction symbolique. Puis, à partir du cas de la firme CS2 and Partners, on illustrera les fonctions de mise en récit de ces images, en montrant combien elles constituent une ressource pour les firmes en termes de construction d'une «façade institutionnelle » (Goffman, 1973) convaincante, en particulier vis-à-vis des clients des cabinets de conseil et des candidat-e-s au recrutement. Enfin, on soulignera les normes corporelles et l'esthétique du groupe à la fois mobilisées et produites par ces supports, les injonctions qu'elles adressent aux salarié-e-s, ainsi que les différenciations et inégalités de genre qu'elles induisent.

\section{Encadré 1 : méthodologie}

La recherche doctorale s'est appuyée sur différents terrains : séquences ethnographiques dans plusieurs entreprises de conseil implantées en France ainsi que dans le syndicat patronal SYNTEC, 72 entretiens biographiques, une enquête 
quantitative auprès de 1637 consultant-e-s dans 23 firmes et des travaux à partir de sources historiques.

L'article s'appuie principalement sur un travail iconographique et mobilisera plus particulièrement deux groupes de ressources photographiques / iconographiques - celles d'un bureau d'organisateurs conseils des années 1950 et celles d'un cabinet de conseil en management contemporain.

- Le premier corpus s'appuie sur les numéros du journal professionnel édité par la firme de l'ingénieur conseil Paul Planus (L'Organisation Paul Planus à ses Amis (OPPSA)), parus d'octobre 1956 à décembre 1961.

- Le second corpus est composé du matériau visuel consulté sur le site internet de la firme CS2 and Partners entre Août 2012 et mai 2015, complété de publications commerciales du même cabinet. (Encadré 3).

Si l'on privilégie ici l'approfondissement de deux cas, la recherche s'est appuyée plus largement sur un corpus de ressources visuelles mises en ligne (photos et vidéos), sur les sites internet de 5 cabinets de conseil en management ${ }^{1}$, faisant partie des firmes enquêtées durant la recherche.

\section{Mettre en scène l'expertise des années 1950 aux années 2010}

5 Les efforts du groupe des consultants pour mettre en scène leur expertise sont à resituer historiquement par rapport à la structuration relativement récente de cet espace professionnel et marchand. C'est en effet au début du XXème siècle, notamment avec l'influence des ingénieurs tayloriens américains, qu'émerge en France, ce métier nouveau qui va accompagner les transformations des structures du capitalisme occidental: mouvements de concentration du capital, développement de la dualité patron, managers, salariés (Moutet, 1997). La propagation de leurs techniques de travail a lieu en France, à l'initiative de la Puissance Publique, à la fin de la $1^{\text {ère }}$ Guerre Mondiale, pour réorganiser, selon les méthodes tayloriennes les usines d'armement. Puis les premiers cabinets, qui resteront peu nombreux jusqu'à la fin des années 1950, vont se développer avec du personnel local masculin $\mathrm{f}$, composé en partie d'ingénieurs formés dans de grandes écoles, en partie d'anciens ouvriers ayant par exemple suivi un cursus au Conservatoire des arts et métiers (Henry, 2012).

6 Le groupe professionnel ne s'institutionnalise pas, en partie parce que le patronat s'oppose à une intervention de l'Etat dans la régulation de ce marché. Deux tentatives de création d'un ordre professionnel des organisateurs conseils échouent dans les années 1940-1950) (Henry, 2006). Le choix du marché plutôt que la solution « d'une profession instituée " apparaît finalement très lucratif, si l'on en juge la croissance extrêmement forte du volume d'affaires produit par l'ensemble de l'espace (Thine, Lagneau-Ymonet, Denord, Caveng, 2013). Cette expansion s'accompagne d'un recours d'abord limité, puis plus conséquent à partir des années $1970^{2}$, à une main d'œuvre féminine, pour atteindre 37 à $40 \%{ }^{3}$ sur la période contemporaine. 
Encadré 2 . L'Organisation Paul Planus à ses Amis. Quand un cabinet des années 1950 fait sa promotion

L'Organisation Paul Planus à ses Amis (OPPSA) constitue une source particulièrement intéressante, à la fois pour appréhender les activités et préoccupations d'un cabinet d'organisateurs conseils au tournant des années 1950-1960, mais aussi pour observer la place qu'y occupent les illustrations, dessins humoristiques, photographies. Sa période de parution couvre cinq années, d'octobre 1956 à juin 1961, dans un contexte de forte croissance de l'activité du cabinet ${ }^{4}$. Dans l'éditorial du premier numéro («La Tribune du Patron »), Paul Planus livre les objectifs de ce journal destiné aux « amis » du cabinet, c'est à dire principalement à ses clients passés et futurs. Il s'agit de " garder le contact », d'entretenir les liens, pour développer les affaires.

La structure des dix numéros - dont la périodicité de sortie est variable - est relativement identique :

Outre l'éditorial du fondateur du cabinet, y sont publiés les biographies professionnelles de certains collaborateurs, les différentes missions réalisées, les changements dans l'organisation du bureau. D'un numéro à l'autre, certains « chantiers » font l'objet d'une présentation plus détaillée. Ponctuellement, la revue commerciale de l'Organisation Paul Planus livre aussi des informations sur le taux de croissance de l'effectif, la formation interne des ingénieurs, les voyages d'étude, réalisés notamment aux Etats-Unis ou le lancement de nouveaux types d'intervention dont le journal fait la promotion. Elle a recours à des photographies individuelles ou collectives présentant les ingénieurs du cabinet, en séance de travail ou prenant la pose, qui accompagnent en général les biographies des collaborateurs. (voir photographie 1 : Paul Planus dans son bureau; photographie 2 : Jean de Sauville). De nombreux dessins souvent humoristiques - (voir illustrations 1 et 2) - illustrent de façon métaphorique le travail, la relation avec les clients ou les questions relatives au personnel.

Dès les premières interventions des ingénieurs conseil en organisation apparaît nettement la question de la légitimité fragile des expertises relatives à l'organisation. Le besoin d'acquérir une forme de stabilité « juridictionnelle » (Abbott 1988) et d'établir la crédibilité de ces savoirs apparaît clairement dans les publications indigènes à visée mi-savante, mi-prescriptive et publicitaire, qui commencent à paraittre en France dès les années 1920, et dont le journal de la firme de Paul Planus est un exemple intéressant (encadré 2: L'Organisation Paul Planus à ses amis. Quand un cabinet des années 1950 fait sa promotion.)

De fait, compte tenu de l'absence de clôture institutionnelle, les représentations du groupe professionnel à travers par exemple des associations comme le Comité National de l'Organisation Française (CNOF) sont assurées par des firmes, qui, les unes et les autres, poursuivent des objectifs partiellement contradictoires : représenter et légitimer le groupe dans sa globalité, tout en s'en distinguant et en construisant sa propre réputation ${ }^{5}$. 
Illustration 1. Dessin humoristique de la page «Chantiers »

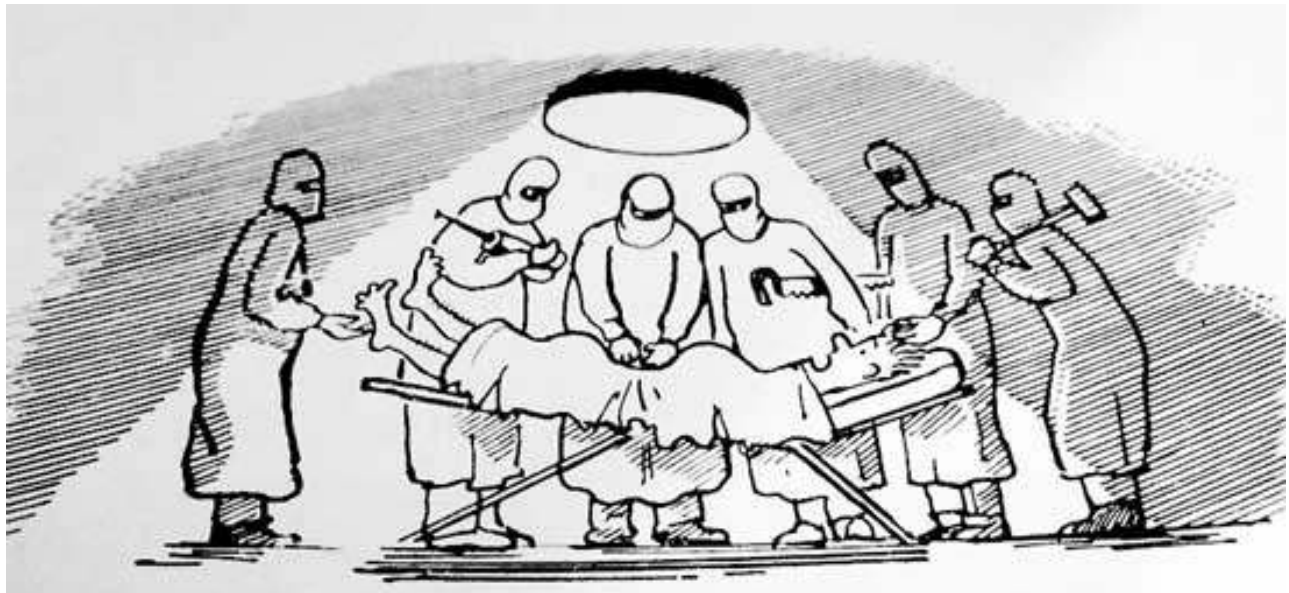

\section{Chantiers terminés.}

Parue dans I'OPPSA

9 Comment s'y prennent les firmes dans cette mise en scène et ce processus de légitimation? Qu'on s'intéresse aux firmes pionnières ou aux firmes contemporaines, on constate un soin particulier à la mise en signe de la qualité du " personnel expert » qui est affecté aux missions réalisées dans les organisations clientes (dès la phase d'émergence, le conseil en organisation s'adresse à la fois à des entreprises privées, à l'administration d'état, aux organisations du tiers secteur).

Illustration 2. Publicité de la firme Paul Planus

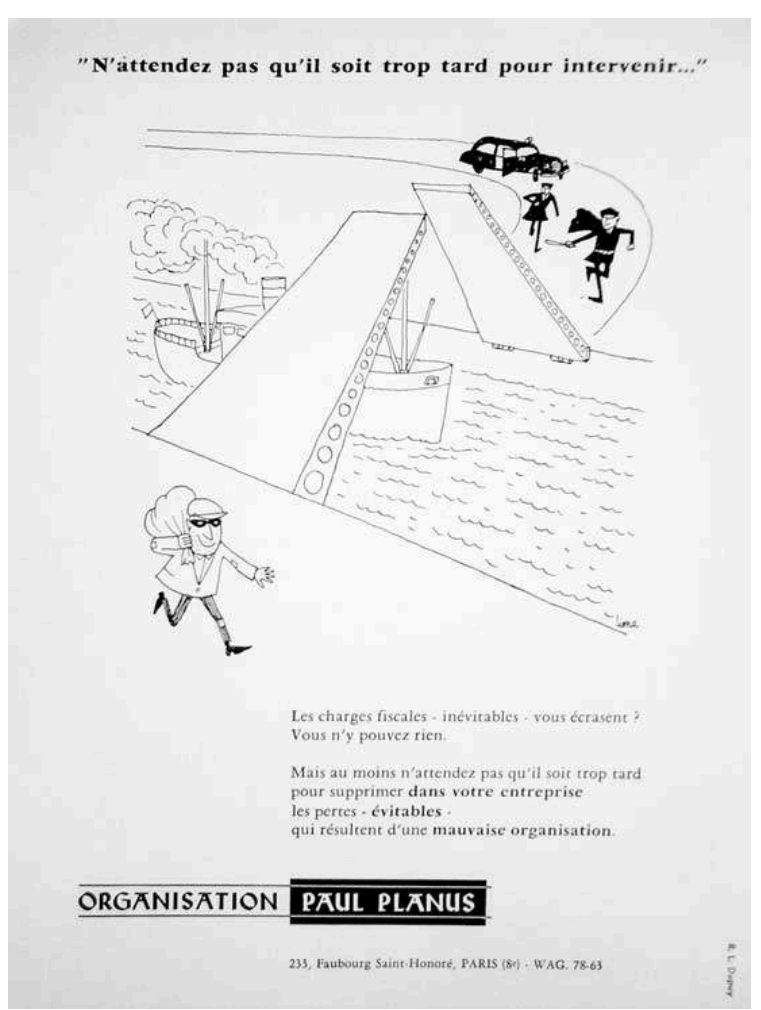


10 Dans le cas du corpus d'images et de textes produits par la firme de Paul Planus, différents procédés alimentent la rhétorique professionnelle. Tout d'abord, la célébration d'individualités, dont le parcours académique et professionnel est présenté avec soin.

11 S'agissant des portraits, ceux-ci sont individuels, présentant les ingénieurs dans une pose figée, offrant l'esquisse d'un sourire, conformément aux normes photographiques de l'époque (voir l'exemple du portrait de l'ingénieur Jean de Sauville). Les biographies professionnelles qui accompagnent les photographies s'emploient à revenir sur les étapes de la carrière des experts présentés ; elles célèbrent des valeurs professionnelles telles que le mérite individuel, l'élitisme et l'anti conformisme. Ainsi, le fait même de célébrer au fil des bulletins, les trajectoires professionnelles forcément réussies ou intéressantes des collaborateurs, montre toute la valeur accordée à des individus qui se distinguent par des efforts spécifiques, à certains égards exceptionnels.

Photographie 2. Paul Planus dans son bureau

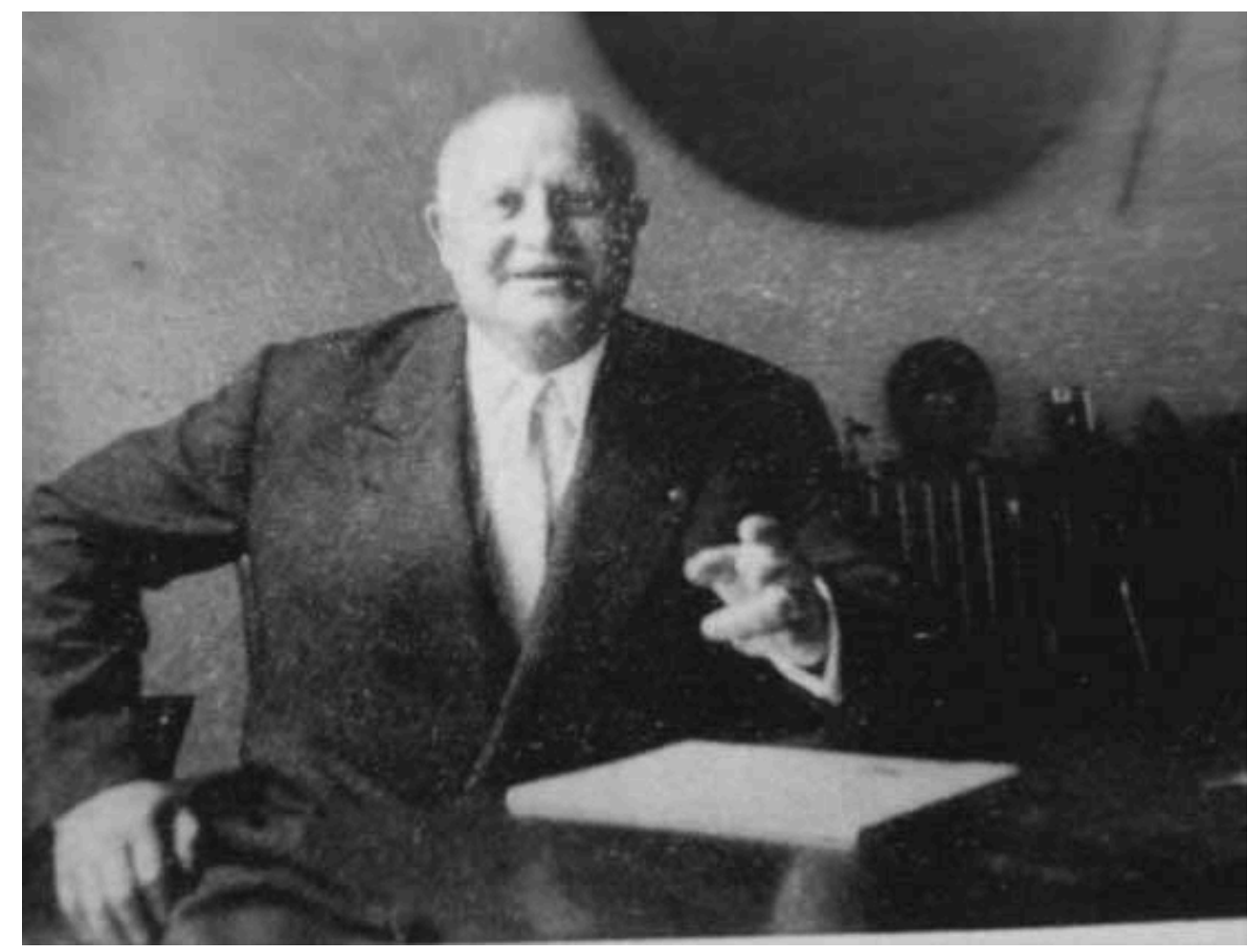


Photographie 3. Portrait de Jean de Sauville

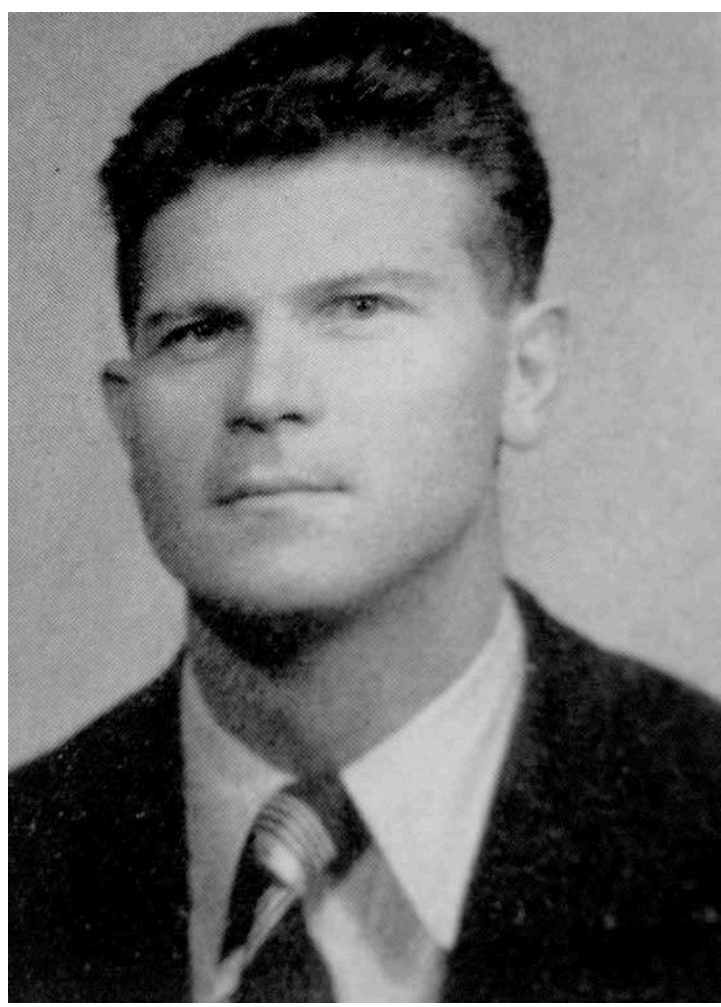

L'élitisme est également un trait marquant des biographies des collaborateurs de Paul Planus : il transparait particulièrement dans le souci constant d'énoncer les rangs, les classements et les distinctions obtenues par les individus dans leurs parcours scolaires, sportifs ou militaires. Enfin, le choix de valoriser, dans les récits, les anecdotes montrant le caractère indépendant et innovant, parfois rebelle et critique des ingénieurs portraiturés, fournit l'occasion d'affirmer une posture anticonformiste. Par exemple, l'ingénieur principal Jean de Sauville est volontiers dépeint comme réfractaire à la routine et préférant sortir des sentiers battus. Non sans un certain goût du second degré, le biographe de Sauville présente d'ailleurs sous cet angle le choix de ce dernier de faire Polytechnique, et d'abandonner une " carrière » littéraire à laquelle il semblait plutôt destiné.

"Après de brillants succès dans les baccalauréats littéraires, il décide, pour ne pas suivre les conseils de ses professeurs de lettre, de présenter Polytechnique. ${ }^{6}$.

Les textes célèbrent par ailleurs volontiers l'anti conformisme, voire l'état d'esprit aventureux, d'experts nomades, allant de "chantier» en "chantier» (jargon de l'époque pour désigner des missions de conseil), tranchant avec l'absence de fantaisie et l'austérité des portraits. Apparaît ainsi progressivement le portrait de l'ingénieurconseil en organisation idéal : à la fois homme de terrain et « matheux ».

En revanche, les illustrations présentent une tonalité tout à fait décalée et souvent humoristique pour décrire certains aspects du métier. De fait, si beaucoup des dessins présentés dans l'OPPSA empruntent l'analogie et la comparaison avec d'autres métiers pour évoquer la posture professionnelle des consultants, les experts du conseil sont représentés sous les traits caractéristiques d'autres groupes professionnels (médecins, policiers, ...), l'évocation relève souvent de l'autodérision : les experts de l'organisation sont par exemple représentés en chirurgiens peu compétents opérant un malade non 
endormi, armés d'outils effrayants (scie, chignole ...). De même, une illustration en contrepoint de la présentation des différents «chantiers » de la firme représente des hommes en costumes, certains en train de se noyer dans un fossé, pendant que d'autres, sur une berge surélevée, les « repêchent » avec des cordes et des harnais (voir illustration 3). La berge porte les initiales de la firme de Paul Planus « O.P.P. », le dessin apparaissant comme une allégorie du métier de conseil qui consiste à porter secours à des organisations en train de sombrer. Toutefois, faute de légende, les intentions du dessinateur peuvent prêter à confusion: les personnes en train d'être secourues pourraient tout autant représenter des collègues organisateurs "pataugeant » dans une mission difficile, et obtenant l'aide d'autres membres du cabinet.

Illustration 3. Dessin humoristique de la vie des « chantiers »

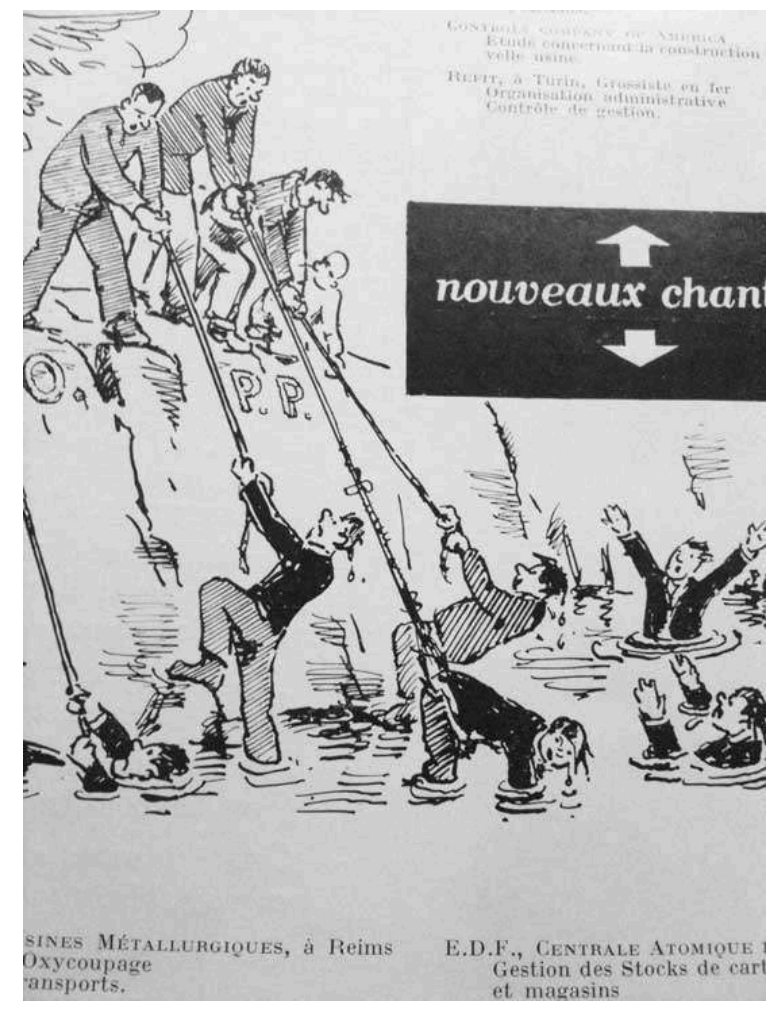

Parue dans I'OPPSA

Quoi qu'il en soit, ces dessins insistent sur la dimension relationnelle du travail, la place des interactions avec les clients, ainsi que sur des valeurs de coopération et d'entraide. Ils renvoient fréquemment à la métaphore du sauvetage, insistant sur la dimension active des interventions des ingénieurs conseils. Ils relèvent au passage que la figure de l'expert légitime est une figure sexuée masculine. L'opposition entre le monde du terrain et des experts et le monde sédentaire de la vie domestique - est d'ailleurs évoquée et mise en image, puisque le journal de l'Organisation Paul Planus réserve une rubrique à la vie privée des ingénieurs ("Scènes de la vie privée de l'organisateur »), qui relate surtout les mésaventures des épouses des organisateurs et qui est illustrée par un dessin figurant Pénélope sur son métier à tisser (voir illustration 4). 
Illustration 4. Pénélope sur le métier à tisser.

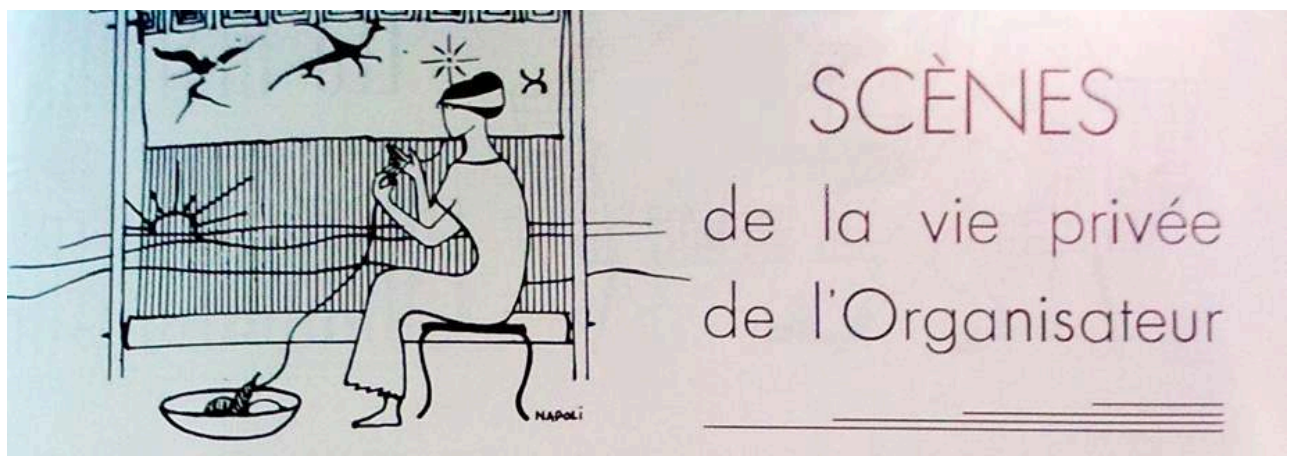

Parue dans l'OPPSA

La tonalité généralement humoristique de nombreuses illustrations de l'OPPSA peut certes interpeller, dans un contexte où les ingénieurs conseils en organisation cherchent à asseoir leur légitimité. Cette tonalité ne trouve pas son équivalent dans les représentations contemporaines du conseil diffusées par les cabinets. Dans l'étude systématique comparée de plusieurs sites internet les présentations trop "réalistes " ou iconoclastes du quotidien du consultant font long feu : après s'être livré à une sorte d' "opération-vérité », par le biais d'un court métrage humoristique - on y voit entre autres un des associés du cabinet travaillant, assis à même le sol, son ordinateur portable sur les genoux, dans un train bondé, un vendredi soir, de retour d'une mission - les dirigeants d'un des cinq cabinets étudiés sur le plan iconographique et visuel, semblent s'être ravisés rapidement, ne laissant la vidéo en ligne sur le site institutionnel que quelques semaines.

\section{Produire des façades convaincantes}

Les sites institutionnels des firmes contemporaines permettent de repérer la poursuite de cette mise en signe de l'expertise avec trois catégories d'images qui coexistent : une présentation stylisée des individus et notamment des partners - qui forment le haut de la pyramide des entreprises de conseil où les individus sont inscrits dans un système de grades et des principes de gestion de carrières élitistes dits up or out (promotion ou éviction) ; des images présentant le collectif expert - soit dans des activités laborieuses - réunions de travail, réflexions collectives, soit lors de rencontres festives; le décor des bureaux de la firme, qui peut être le « sujet » de la photo (le site de CS2 and Partners montre ainsi une dizaine de photos de ses locaux). Cette mise en signe peut être abondante : pas moins de 142 photos sur le site de CS2 and Partners (voir encadré 3). 


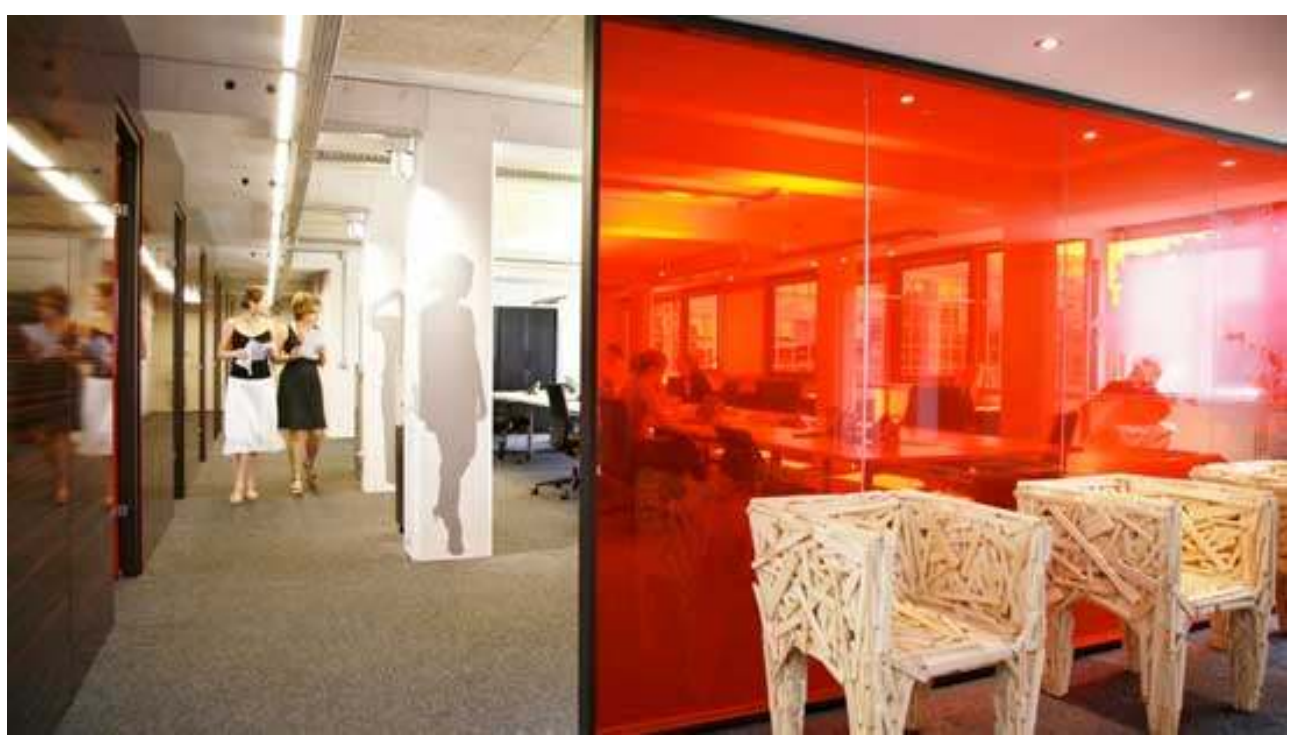

\subsection{Une importante vitrine symbolique}

18 Le site internet, support de l'analyse, présente un exemple intéressant pour approfondir ce que produit, sur le plan symbolique, ce matériau iconographique. CS2 and Partners est ce que le jargon professionnel a baptisé un spin off, c'est à dire une entreprise dont l'équipe de dirigeants et fondateurs est composée de transfuges, partis avec des collaboratrices et collaborateurs d'un grand cabinet de conseil réputé. La firme a connu une croissance rapide : fondée en 2001, elle compte 130 consultants en 2010-2011 et 350 personnes en 2015, si l'on tient compte de ses filiales.

Encadré 3 : Le site internet de CS2 and Partners, une importante vitrine symbolique

Abondamment illustré par 142 photos ainsi qu'une dizaine de vidéos, présentant l'ensemble de l'équipe de direction (les « senior partners ", les directeurs associés), certain-e-s consultant-e-s et les locaux de la firme, le site est subdivisé en 4 grandes rubriques : Un métier, Des hommes, Un style, Des carrières, avec pour chacune, des textes dont le contenu argumente les spécificités et l'excellence de CS2.

Elément central de la rhétorique professionnelle : « la transformation » dont le cabinet se présente comme un agent actif au service des entreprises et de la société. Un senior partner décline son identité: « (...) je suis « fabricant de transformation » au service de la performance des entreprises $»^{7}$.

Présentés sans commentaires spécifiques, les clichés côtoient de petits articles qui se répondent pour définir le style et revenant sur une (forte) culture d'entreprise (Culture), une expertise acquise et maîtrisée comme un art (L'Art de la Transformation), et la transmission généreusement orchestrée de ces savoir-faire (La prise de parole). Certaines photos véhiculent l'idée du nomadisme, par ailleurs vanté dans le profil du Consultant transformateur : " Au quotidien, il est présent chez le client, proche des équipes ", animé par une " passion pour l'exploration de nouveaux horizons ". Le collectif est par ailleurs mis en scène, avec des photos de 
groupes, en réunion ou saisis lors d'échanges informels. Collectif dont la convivialité et la simplicité sont célébrées en montrant les consultants installés de manière spartiate, à même les marches en bois de l'escalier sur lequel ouvre le hall. L'idée de « singularité » (" un modèle responsable et singulier ») est largement appuyée, par la métaphore de la « signature » (« Nos clients nous reconnaissent une signature », " une signature unique »), renvoyant à l'intuitu personae des relations cabinet-clients et à celle d'unité du collectif. « Nos consultants sont le reflet de notre singularité » témoigne un Partner.

Elle donne à voir les investissements à la fois matériels et symboliques opérés par une nouvelle organisation pour construire sa réputation : on observe ainsi chez CS2 and Partners la recherche de conformité à certains éléments de l'ethos professionnel - en revendiquant l'inscription dans une «dynastie professionnelle $»^{8}$ et le désir de faire émerger "un style", une "signature" particulière. Dans cette stratégie de légitimation, le site internet institutionnel apparaît donc très investi avec une présentation didactique des interventions, des consultants, des locaux, des productions professionnelles et académiques. Il manifeste de façon exemplaire la fonction de vitrine symbolique que revêtent ces espaces virtuels, ou pour le dire comme Erving Goffmann, de «façade institutionnelle», système symbolique qui "pour fonction normale d'établir et de fixer la définition de la situation qui est proposée aux observateurs » (Goffman 1973 :29). La façade collective est produite par un ensemble de signes qui construisent une impression cohérente (encadré 3).

\subsection{Une esthétique soignée}

Les photos très nombreuses présentées sur le site internet de la firme témoignent d'un même souci esthétique et veillent à mettre en valeur des individus et des collectifs en situation, souvent pris «sur le vif» dans les locaux de la firme, locaux qui participent activement à la production d'une impression d'ensemble (Boni-Le Goff, 2015).

Photographie 5. CS2 and Partners

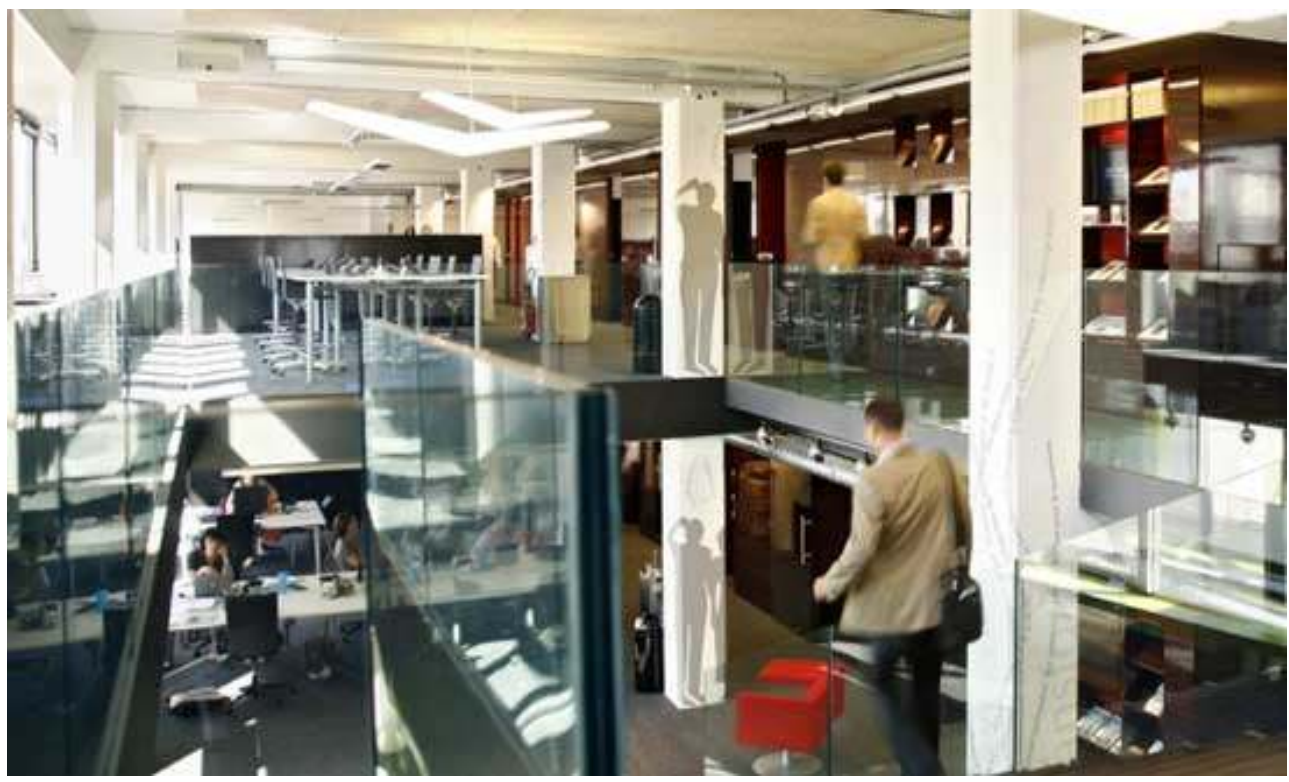


21 L'usage récurrent de certains procédés, photographies montrant par exemple des silhouettes floues et en mouvement ou prises en "grand angle », offrant une vision très large sur les vastes bureaux ouverts des locaux en mezzanine de la firme - contribuent à véhiculer notamment l'idée de changement et de modernité, faisant écho au discours que tient la direction de l'entreprise sur sa " raison d'être ». L'esthétique très soignée de tous les clichés mis en ligne (souci de l'éclairage, cadrage professionnel, ...) témoigne également d'une volonté de contrôle des impressions et de mise en valeur symbolique par l'image. Par ailleurs, la tenue des consultant-e-s dont les portraits sont présentés, signale le souci de la présentation de soi - nous reviendrons plus loin sur l'hexis vestimentaire et la tenue corporelle. Chacun-e présente une "façade personnelle» (Goffman, 1973) qui participe de manière ajustée à la façade collective.

Comme d'autres cabinets étudiés, CS2 and Partners ajoute toutefois aux présentations formelles, de petits reportages (sous forme de vidéos ou de photo-reportage), comportant récits ou témoignages sur le métier et les pratiques du cabinet. C'est le cas d'une vidéo de présentation mise en ligne sur le site de CS2 and Partners, qui présente de manière scénarisée, trois situations de travail « typiques » du conseil, et confronte pour chacun de ces cas de figures, deux manières de faire le travail, deux «styles de conseil $»$. Le spectateur comprend rapidement que le cabinet défend et incarne le « deuxième style » dont le film s'emploie à démontrer la modernité et l'efficacité.

Quels sont les publics auxquels une telle mise en image peut s'adresser? Les destinataires de cet investissement symbolique sont multiples : il s'agit à la fois des clients (et prospects) de l'entreprise, des firmes concurrentes et des candidat-e-s à un recrutement. Cette dernière catégorie constitue un enjeu très important pour un cabinet comme CS2 and Partners, dans la mesure où la qualité des recrutements est un point de vigilance particulier des directions d'entreprises acheteuses de conseil.

24 Dans la fraction la plus prestigieuse de l'espace du conseil en management, dans laquelle CS2 and Partners souhaite être reconnue, les cabinets se livrent à des luttes concurrentielles vives sur le marché des diplômé-e-s des grandes écoles. Condition de maintien du prestige, la capacité à recruter les "meilleurs profils» dérive d'une réputation globale, construite de façon composite et à laquelle participe l'image générale véhiculée par l'entreprise via des investissements matériels et symboliques ${ }^{10}$. Co fondateur de CS2 and Partners, Philippe ${ }^{11}$ insiste sur la construction d'une culture d'entreprise cohérente, qui doit pouvoir être déclinée à la fois dans les choix concrets, architecturaux, d'aménagements des lieux comme dans les images produites. Il revient sur l'aménagement des bureaux de l'entreprise, décor largement mis en image sur le site de la firme, et sur une mascotte (un petit personnage imaginaire en plastique aimanté) conçue et reproduite à un millier d'exemplaires au moment du $10^{\text {ème }}$ anniversaire de l'entreprise.

«L'idée, c'est ... on a besoin de symboles, on a besoin de rites, c'est ça qui fait la

force des sociétés. »

25 En pastichant le film «Le fabuleux destin d'Amélie Poulain $»^{12}$, la direction de CS2 and Partners organise le «tour du monde » de sa mascotte, la faisant prendre en photo par des complices dans différents endroits du globe. Des étudiants d'HEC en mission humanitaire lui font gravir un des plus hauts sommets du monde, ce qui souligne au passage combien ces investissements symboliques de l'entreprise sont en particulier destinés notamment au public des futur-e-s candidat-e-s. 
Les sites internet des sociétés de conseil présentent d'autant plus d'enjeux qu'ils sont très fréquemment visités par les candidats au recrutement pour y puiser des informations sur l'entreprise et préparer le cycle d'entretiens qui fait partie du processus de sélection. Comme pour tous les autres sites de cabinets de conseil analysés pendant l'enquête, celui de CS2 and Partners, a prévu des rubriques qui sont clairement pensées pour répondre aux questions des candidat-e-s : par exemple, la rubrique « Des carrières" comporte plusieurs entrées destinées à de futur-e-s candidat-e-s: «Etudiants et Jeunes diplômés» (avec des titres comme «Devenir consultant transformateur", décrivant le processus de formation des jeunes recrues) ou "Consultants Expérimentés " (et des sous rubriques comme «Notre conception de l'excellence »). Un «Espace Ecoles Partenaires» est par ailleurs réservé à des écoles d'ingénieurs et de commerce très convoitées (HEC, ESSEC , ESCP, Polytechnique ...), qui constituent un vivier privilégié de recrutement pour le cabinet: chaque école est "parrainée " par deux ancien-ne-s élèves, devenu-e-s consultant-e-s de CS2 and Partners, dont les portraits souriants (face et haut du buste) sont présentés avec indication de leur prénom («Julien, ESSEC 2014 », « Manon, HEC 2012 »). L'idée d'intuitu personae est là encore affirmée. Sont également annoncées dans ces rubriques les interventions académiques de senior partners qui donnent des enseignements ou des présentations sur le conseil sur les campus des différentes écoles partenaires ${ }^{13}$.

On a donc dans cette partie, évoqué une façade qui se construit et livre une forme de représentation théâtrale. Façade qui alimente la légitimité du groupe. A travers la profusion de photographies et de vidéos, CS2 and Partners déploie ainsi d'importants efforts pour produire une façade institutionnelle maîtrisée : l'image véhiculée est celle d'un cabinet qui tout à la fois, se conforme aux valeurs de l'ethos professionnel et du logos gestionnaire (Boussard, 2008) et tente de se distinguer, en forgeant "un style", "une signature». Les images ainsi produites de l'expertise et des experts "en situation » participent à une mise en récit professionnelle très élaborée, que les textes en ligne dans les différentes rubriques contribuent aussi à construire. Mise en récit qui s'adresse d'abord aux candidat-e-s au recrutement et dont un des buts est de démontrer le prestige et l'élitisme d'un métier aux contours et contenus restant relativement flous.

28 A bien y regarder, les destinataires de ce discours mis en image ne se limitent toutefois pas aux différents publics identifiés, clients, candidat-e-s, ... A certains égards, les images produites méritent d'être examinées sous l'angle de leur portée normative, pour saisir « ce qu'elles font » aux consultant-e-s elles/eux-mêmes.

\section{Ce que les images font aux professionnel-le-s}

29 Les photos à la fois collectives et individuelles mises en ligne contribuent à produire et activer des normes corporelles, vestimentaires, de la figure du consultant légitime, normes qui opèrent des injonctions à l'endroit des professionnel-le-s.

\subsection{Des normes corporelles et vestimentaires genrées}

30 Si on effectue un retour sur les photos individuelles ou collectives des consultant-e-s, celles-ci proposent des figures très homogènes des experts de la gestion. Le cadrage présente le visage et parfois le buste, de face ou trois-quart face, l'arrière-plan étant 
totalement neutre ou permettant juste de deviner quelques éléments de décors (étagères, fenêtres...). De façon systématique, les consultants "ont pris la pose " et fixent l'objectif, le visage portant un sourire franc ou esquissé. En dépit d'une variété affichée, il se dégage de l'observation une impression générale d'homogénéité, qui tient en particulier à certains traits et attributs communs des protagonistes, une sorte de "signature" des façades personnelles des experts: entre autres, la minceur (à l'exception de quelques rares partners légèrement bedonnants) et le maintien (assis ou debout, aucun consultant n'est " avachi »). Les vêtements portés contribuent également à véhiculer l'impression d'homogénéité : hommes en costumes sombres (éventuellement «en chemise » blanche); femmes la plupart du temps en vestes de tailleurs et chemisiers clairs. Les variations sont ténues : port ou pas de la cravate par exemple, le premier cas étant toutefois le plus fréquent. Les accessoires, y compris féminins, se révèlent relativement discrets (belles montres, bijoux peu voyants).

31 Ces différentes images dessinent une figure de l'expert légitime qui apparaît clairement masculine : d'une part sur le plan numérique, les photos présentent majoritairement des hommes; d'autre part, sur le plan symbolique, car la figure de l'expert légitime apparaît construite sur la base d'un référentiel masculin qu'il s'agisse de codes vestimentaires (la veste sombre par exemple) ou de l'hexis corporelle. La comparaison des portraits des consultants hommes conduit à observer des pratiques esthétiques, corporelles, conformes, pour le dire comme Paul Lagneau-Ymonet, à une « masculinité d'affaires » ou à ce que Catherine Achin, Elsa Dorlin et Juliette Rennes désignent comme une "virilité privilège» (Achin, Dorlin, Rennes, 2008). Virilité caractérisée par le contrôle des émotions (Elias, 1973) et du corps (musculature effacée, minceur, maintien, ...) et par l'idée d'ascèse (relatif dépouillement des tenues).

On peut revenir à propos de ces observations, sur les remarques de Paul LagneauYmonet (2007) s'agissant d'un répertoire de "masculinité d'affaires", produit historiquement par les agents de change et les professionnels de la finance, et fonctionnant comme une ressource dans des stratégies de légitimation et de (re)valorisation d'un groupe professionnel. Tout en rappelant la force et la prégnance des références viriles dans le domaine de la finance et des affaires, P. Lagneau-Ymonet montre que la résistance de ce référentiel s'agissant des marchés financiers tient pour partie à la quête d'une respectabilité, loin d'être acquise pour des activités assimilées aux jeux de hasard : «Tout au long du (XIXème) siècle, les agents ont eu à se démarquer des repoussoirs qu'étaient la maison de jeu, le lieu de perdition, et la prostituée, figure du désordre et de l'imprévoyance. » (Lagneau Ymonet 2007 :115). Cette observation fait évidemment écho aux questions évoquées en première partie sur la légitimité fragile du groupe des consultants.

Ces différentes normes corporelles et d'apparence qui sont donc articulées plus largement à l'ethos professionnel (et participent à en activer et symboliser certaines valeurs), apparaissent à la fois comme des contraintes et des ressources pour l'exercice du travail relationnel avec les clients. Le travail ethnographique a permis de constater que ces normes étaient activement mises en jeu par les consultants, et qu'elles formaient un référentiel pour le travail d'incarnation de l'expert légitime, en particulier dans les interactions avec les clients. On peut faire le lien avec le travail réalisé par Alexandre Mathieu-Fritz sur les huissiers, et pour lesquels également se pose la question de la construction de la crédibilité dans les interactions et le travail dit «d'exécution » (Mathieu-Fritz, 2003). Comme dans le cas des huissiers, les consultant- 
e-s peuvent souvent comparer leur travail relationnel à « un théâtre ». L'ethnographie relève des transactions multiples qui sous-tendent cette activité dramaturgique chez les consultants: transactions corporelles, vestimentaires, vocales, tout autant que discursives, pour produire une impression cohérente et conforme aux attentes socialement construites s'agissant de l'expert légitime. Les consultant-e-s travaillent l'art oratoire souvent à l'occasion de sessions animées par des collègues plus anciens, en position de management. Lors de l'observation participante dans une des firmes étudiées, on a pu suivre une semaine de "stage résidentiel» destinée aux jeunes recrues, initiées à une certaine présentation de soi, à la prise de parole et aux techniques de gestion des réunions.

Le passing, concept développé par Harold Garfinkel (2007), apporte un outil intéressant pour penser l'ensemble de ces transactions, en partie réflexives, qui permettent « dans des conditions socialement construites (d') acquérir le droit à vivre selon un statut ». Ce concept permet d'envisager les enjeux, pour tout-e professionnel-le, du travail relationnel: pour les consultant-e-s, il s'agit d'un passing expert, qui permet d'être accepté-e comme « digne représentant » de sa profession et d'être qualifié-e dans et par l'interaction.

Dans ces passings experts, les normes corporelles et vestimentaires, en partie activées dans les nombreuses images du travail qui circulent, sont comme on l'a vu, non neutres sur le plan du genre. Elles sont problématiques pour les consultantes, qui rencontrent des difficultés particulières pour incarner l'expert-e légitime. Dotée d'une certaine ancienneté, Alix ${ }^{14}$ résume le dilemme des femmes consultantes pour se faire accepter " dans un métier où (...) on théâtralise beaucoup.»

«Et donc, dans un métier où de fait, on théâtralise beaucoup (...) Il ne faut pas faire exactement la même chose (NDLA : que les hommes), parce que, dans ce cas-là ... c'est ça qui est compliqué, hein? Parce qu'on dit ça c'est une femme qui se comporte comme un homme et ça, c'est perçu négativement. Mais une femme trop effacée, trop timide, c'est perçu comme une « nobody ». »

Comme d'autres consultantes, elle évoque à ce propos la lancinante question vestimentaire, qui, sous un aspect prosaïque et anodin, révèle les normes corporelles et d'apparence genrées en vigueur dans l'espace professionnel et plus généralement associées à la figure des managers. Dans son étude sur les processus d'appréciation de la conformité au groupe de référence "cadres", Marie-Thérèse Duflos-Priot (1976) analyse comment se construisent la catégorisation et la reconnaissance à travers des "signes" distinctifs vestimentaires, corporels, pour des observateurs issus de différents milieux sociaux : à la base du jugement de conformité (les observateurs se prononcent sur la probabilité d'appartenance au groupe des cadres de plusieurs hommes pris en photo), se trouvent des attentes socialement formées qui se réfèrent à une définition stabilisée de l'apparence "normale ", habituelle du membre du groupe "cadres » et la reproduisent. Tentant elles aussi de s'ajuster à cette définition stabilisée, les consultantes rencontrées pendant l'enquête, comme celles observées sur les portraits mis en ligne sur les sites internet, usent par exemple de façon majoritaire d'une pièce vestimentaire masculine, la veste de costume, pour agrémenter des tenues, qui par ailleurs empruntent à des éléments relativement ritualisés du vestiaire féminin (jupes ou robes, chaussures à talons hauts, ...). Elles évoquent les incertitudes d'un "double standard» (Wajcman 1998) auquel elles sont en permanence confrontées: paraître "professionnelles", rester "féminines». Les cas de disqualification de l'expertE, liée à son apparence, à sa tenue, sont fréquents. 


\subsection{Une représentation stéréotypée des rôles et de la division du travail}

37 Si les images contribuent à produire et reproduire la figure masculine de l'expert, elles peuvent également, tout en mettant en signe une relative mixité femmes/hommes, véhiculer dans la représentation des collectifs, une image relativement stéréotypée de la division sexuelle du travail et des rôles dans le conseil.

L'analyse plus détaillée de la façon dont sont montrées les consultant-e-s au travail sur le site internet et sur les photos de certaines plaquettes publicitaires de CS2 and Partner, est significative sur ce point. Les mises en situation professionnelles scénarisées tendent à mettre en scène des interactions structurées par des oppositions genrées qui rejoignent les observations de Carole Brugeilles, Isabelle Cromer et Sylvie Cromer (2002) à propos de la littérature et des albums destinés aux enfants. Celles-ci notent: « (...) la construction d'identités et de rapports sociaux de sexe est bien au cœur de la problématique des albums avec l'élément central que sont les personnages. Ces représentations s'élaborent, non sur la base de stéréotypes immédiatement repérables, mais de manière fine et complexe, à partir d'un ensemble de variables : le sexe, l'âge, le rôle (personnage principal, secondaire, d'arrière-plan) (...) les activités professionnelles du personnage » $(2002: 289)$

L'observation systématique des onze photos d'une brochure présentant la philosophie et les méthodes d'intervention du cabinet (éditée en 2012), conduit à remarquer une différenciation genrée des rôles entre consultantes et consultants. S'ajoutant à la supériorité numérique masculine ${ }^{15}$, celui qui incarne le personnage central ${ }^{16} \mathrm{du}$ «consultant-en-situation » est un homme dans 8 photos sur 10, alors qu'il s'agit d'une femme dans seulement une des 10 photos et qu'il est incertain dans la dernière ${ }^{17}$. Ce personnage principal prend l'initiative et la parole, avec une position corporelle qui renforce symboliquement son statut dominant (position haute, sur une marche ...) ; les regards sont tendus vers lui. En nombre limité sur ces photos scénarisant le travail de l'expert, les femmes qui y figurent, sont en définitive surtout cantonnées à des postures conformes à la division sexuée conventionnelle des rôles sociaux (posture d'écoute, regard tourné vers celui qui parle ...).

40 D'une durée d'environ 3 minutes, la vidéo déjà citée qui présente, sur le site de CS2 and Partners, trois situations de travail «typiques » du conseil, et confrontant deux « styles de conseil », illustre également une représentation genrée de l'expert légitime. On constate tout d'abord la domination numérique des hommes dans les deux premières parties (un groupe de quatre individus avec seulement une femme); si dans la troisième partie, celui qui incarne le personnage central du consultant-en-situation, un homme interagit avec deux femmes, l'une occupe une position aisément reconnaissable d'hôtesse d'accueil (jeune femme souriante derrière un comptoir à l'entrée de l'immeuble), l'autre, celle d'une consultante qui semble être plus junior que «leconsultant-en-situation » et placée sous la supervision de ce dernier.

Une domination masculine, symbolique cette fois, apparaît clairement dans l'ensemble des situations présentées dans la vidéo. Dans la première qui représente une réunion chez un client, les «acteurs » qui incarnent les experts (il s'agit de consultant-e-s de CS2 qui ont accepté de participer à ce "docu-fiction») sont deux hommes. Debout, figurant des experts maîtres d'eux-mêmes et engagés dans des explications, ils font face 
à un auditoire passif, assis autour de la table (une femme et un homme). Le second moment du film évoque une réunion où opère la co-production d'idées (on peut imaginer des consultants et des client-e-s). De la même façon dans cet extrait, celui qui prend l'initiative et gère la prise de parole de chacun-e est un homme.

De façon intéressante, si les deux styles de conseil sont opposés quant aux pratiques adoptées, la figure de l'expert est donc toujours celle d'un homme, le même "acteur " jouant le rôle du «mauvais » consultant ( $1^{\text {er }}$ style évoqué dans chaque partie) et du "bon» (second style). Assez caricaturale dans un cabinet caractérisé par une féminisation significative du personnel expert ${ }^{18}$, cette représentation ritualisée du travail donne ainsi un accès saisissant aux stéréotypes de genre et aux processus qui contribuent à les alimenter et les reproduire. Ces stéréotypes sont à rapprocher d'inégalités dans les carrières et d'un plafond de verre persistant dans l'ensemble de l'espace (Boni-Le Goff, 2012).

43 Ainsi, à travers ces deux exemples transparait une vision des rôles sociaux et de la division du travail qui tend à reproduire les frontières de genre. S'il s'agit d'une représentation du travail dont les auteurs ou les commanditaires sont des acteurs situés socialement (ici les photos sont commanditées par les dirigeants du cabinet CS2 and Partners) et qui ne doit pas être confondue avec le réel du travail, ces images éclairent la prégnance de stéréotypes, celle des figures idéal-typiques de l'expert légitime. Elles soulignent les résistances des rapports sociaux de sexe dans l'espace professionnel du conseil. Comme le rappelle Christine Detrez, les photographies « conventionnalisent nos conventions, stylisent ce qui l'est déjà » (Detrez $2002: 151)$. Si les mises en image contemporaines ne versent pas dans des caricatures aussi outrées que celles dont ont fait l'objet les pionnières entrant dans des professions masculines prestigieuses (Rennes, 2013), on assiste au déplacement plus qu'à l'effacement des frontières de genre. Les images continuent à entretenir «l'effet d'évidence des représentations dominantes » (Rennes, $2013: 416)$ du groupe professionnel.

En conclusion, l'analyse éclaire la richesse heuristique d'une analyse des groupes professionnels au prisme des récits iconiques qu'ils produisent sur eux-mêmes. Cette analyse prend un relief particulier, si l'on observe les formes et variations historiques de ces productions ; elle apporte un éclairage original sur les luttes juridictionnelles et la quête de légitimité, dans lesquelles les acteurs des groupes étudiés sont engagés.

L'étude de la mise en images du groupe des consultants a ainsi offert l'opportunité d'observer la place réservée aux images pour un groupe professionnel engagé dans un tel travail de légitimation, qu'il s'agisse des acteurs pionniers dans l'espace émergent du conseil ou d'entreprises contemporaines. L'investissement iconographique et son contenu dessinent la construction d'une façade institutionnelle, qui passe à la fois par la représentation des collectifs et celles d'individus mis en signe de façon stylisée. Tout en véhiculant l'idée de singularité, d'unicité, le discours iconographique célèbre l'inscription dans une certaine tradition et un savoir-faire hérités. Sont à la fois mises en avant la détention collective du savoir et sa mise en jeu dans des relations intuitu personae spécifiques.

L'analyse permet par ailleurs d'envisager les effets des images pour les professionnelle-s, notamment en termes de résistance des frontières de genre. De fait, pour les consultant-e-s, engagé-e-s dans un important travail d'incarnation de l'expert légitime, les normes corporelles et d'apparence, véhiculées et confortées par le matériau photographique sont à la fois des ressources et des contraintes qui reproduisent des 
frontières genrées. Celles-ci sont à relier au maintien d'inégalités de genre, qu'il s'agisse de l'exercice quotidien du travail (notamment les activités relationnelles) ou de la carrière et de l'accès des femmes consultantes aux fonctions dirigeantes.

\section{BIBLIOGRAPHIE}

Abbott A., The system of professions - An essay on the division of expert labor, Chicago, The University of Chicago Press, 1988, 452 p.

Achin C., Dorlin E., Rennes J. (2008) « Capital corporel identitaire et institution présidentielle : réflexions sur les processus d'incarnation des rôles politiques ", Raisons politiques 2008/03, $n^{\circ} 31$, p. 5-17

Boni-Le Goff I. (2012), « Ni un homme, ni une femme, mais un consultant. Régimes de genre dans l'espace du conseil en management », Travail et Emploi, $\mathrm{N}^{\circ} 132$, p. 21-34

Boni-Le Goff I. (2015), « A quoi servent les halls des cabinets de conseil ? Espace, biens symboliques et techniques de gouvernement. ", Genèses, N 99 , p. 48-68

Boussard v., Sociologie de la gestion - Les faiseurs de performance, Paris, Belin, 2008, 203 p.

Brugeilles C., Cromer I., Cromer S. (2002), « Les représentations du masculin et du féminin dans les albums illustrés ou comment la littérature enfantine contribue à élaborer le genre », Population, 2002/2, vol. 57, p. 261-292.

Chandler A. (1988), La main visible des managers, une analyse historique. Paris, Economica

Collinson D.L., Hearn J. (1996), Men as managers, managers as men. Critical perspectives on men, masculinities and managements, London, Sage, $275 \mathrm{p}$

Detrez C. (2002), La construction sociale du corps, Paris, Seuil

Duflos-Priot M.T. (1976), « Paraître et vouloir paraître. La communication intentionnelle de l'apparence », Ethnologie française, VI, pp. 3-4.

Elias N. (1973), La Civilisation des mœurs, Paris : Calmann-Lévy, 342 p.

Foucault M. (2001). Dits et écrits II, 1976-1988. Paris : Gallimard

Garfinkel H., (2007), Recherches en ethnométhodologie, traduction coordonnée par M. Barthélémy et L. Quéré, Paris, PUF, 2007, 473 p.

Gadrey J., Socio-économie des services, Paris, La Découverte, 2003, 123 p.

Géhin J-P. et Stevens H. (dir.) (2012), Images du travail, travail des images, Rennes, PUR.

Goffman, E., (1973), La mise en scène de la vie quotidienne la présentation de soi. Paris, Les Editions de Minuit

Henry, O. (2006), « L'impossible professionnalisation du métier d'ingénieur conseil (1880 - 1954)» Le Mouvement Social, 214, p. 37-54.

Henry, O. (2012), Les guérisseurs de l'économie - Ingénieurs-conseils en quête de pouvoir. Socio-genèse du métier de consultant (1900-1944), Paris, CNRS Editions 
Mathieu-Fritz A., (2003), « La compétence relationnelle dans l'application des décisions judiciaires. Eléments pour une sociologie de l'action des huissiers de justice dans le cadre du recouvrement de créances ", Réseaux 2003/5 ( $\left.\mathrm{N}^{\circ} 121\right)$, p. 173-202.

Moutet, A. (1997), Les logiques de l'entreprise : la rationalisation dans l'industrie française de l'entredeux-guerres, Paris, Editions de l'EHESS

Kerfoot D., Knights D. (1996), « The best is yet to come? The quest for embodiment in managerial work » in D. Collinson and J. Hearn, Men as managers, managers as men. Critical perspectives on men, masculinities and managements, London, Sage, p. 78-98

Lagneau-Ymonet P., (2007) « La masculinité d'affaires. Le cas de la Bourse de Paris (1724-1988) », in R. Revenin, Hommes et masculinités de 1789 à nos jours, Paris, Autrement, 2007, p. 113-127

Rennes J., (2013), « Genre, travail et culture visuelle » in M. Maruani, Travail et genre dans le monde. L'Etat des savoirs, Paris, La Découverte, p. 409-418

Terrenoire J.P., (1985), « Images et sciences sociales », Revue Française de Sociologie, XXVI, p. 509-527.

Thine S., Lagneau-Ymonet P., Denord F. et Caveng R. (2013), « Entreprendre et dominer. Le cas des consultants ", Sociétés Contemporaines, n89: 73-99

Wajcman J. (1998), Managing like a man: women and men in corporate management, University Park, Pennsylvania State university Press

\section{NOTES}

1. Les sites internet des 5 firmes sont accessibles aux adresses suivantes: www.accenture.fr, www.bcg.com, www.nouvellesdonnes.com, www.rolandberger.fr, www.keapartners.com

2. A partir du moment où les femmes ont eu un accès généralisé aux mêmes formations supérieures que les hommes, notamment au système - spécifique à la France- des grandes Ecoles d'ingénieurs et de commerce (Polytechnique, HEC, ESSEC, ESCP, n'ouvrent leurs concours aux femmes qu'au début des années 1970).

3. Sources enquête emploi et enquête 2008 auprès de 23 firmes.

4. Créé en 1924, le développement du bureau de Paul Planus est d'abord lent puisqu'en 1939, il ne compte encore que 19 personnes. Un tournant décisif est pris à la fin des années 1940. En 1957, l'Organisation Paul Planus compte désormais 70 ingénieurs et 6 directeurs. Sources : L'OPP à ses amis ; voir aussi Aimée MOUTET (1997).

5. Fondé en 1925 par des promoteurs du taylorisme, le CNOF est une association animée par les fondateurs des firmes pionnières tels Paul Planus (Moutet, 1997).

6. OPPSA, juin 1957

7. Interview sur le site de CS2, consultation du 15 janvier 2015

8. La présentation de l'historique de la firme tout comme d'autres textes présentant les biographies des partners par exemple, font à de multiples reprises référence à un cabinet de conseil pionnier fondé dans les années 1960 - Bossard Consultants - firme qui a installé sa notoriété et est devenue exemplaire d'un pôle « intellectuel » de l'espace français du conseil. Une majorité des fondateurs de CS2 and Partners a été formée au sein de cette firme qui sera ensuite reprise par la société de services informatiques Cap Gemini en 1997.

9. Vidéo consultée en ligne en août et septembre 2012. Lors d'une nouvelle consultation en décembre 2012, cette vidéo ne figurait plus sur le site.

10. La réputation repose bien évidemment également sur les capitaux scolaires des partners et senior managers du cabinet, capitaux qui sont systématiquement mobilisés dans les biographies 
mises en ligne et qui constituent une hyperbole censée résumer l'excellence des personnes considérées et donc de la firme. Dans le cas de CS2 and Partners, les diplômes des 23 partners, et directeurs associés systématiquement cités, se répartissent de la manière suivante : 5 diplômé-e-s d'écoles de commerce parisiennes (HEC, ESSEC, ESCP), 9 diplômé-e-s d'écoles d'ingénieurs de rand A (Polytechnique, AgroParistech, Mines, Centrale ...), 1 diplômé de SciencePo Paris, 5 diplômé-e-s d'écoles de commerce de province (EDHEC ...), 3 diplômé-e-s (DEA ou DESS) de l'université.

11. 60 ans, Polytechnique, Ingénieur Eaux et Forêts, ancien senior Partner et principal fondateur de CS2, entretien mars 2015

12. Long métrage réalisé par Jean-Pierre Jeunet en 2001.

13. Dernière consultation du site le 16 mai 2015

14. 35 ans, école de commerce parisienne, manager, cabinet de conseil en stratégie, entretien 2011

15. Celle-ci est marquée pour les 10 photos présentant de petits groupes de 2 à 4 personnes, puisqu'on compte 18 hommes et 7 femmes. Pour la 11ème photo qui figure un groupe de consultant-e-s assis sur les marches du grand escalier de CS2 and Partners, apparemment en train d'écouter une conférence, on relève une plus grande mixité numérique : 16 hommes et 13 femmes ( 2 silhouettes dans l'ombre n'ont pas été prises en compte).

16. Je reprends ici la définition que donnent $C$. Brugeilles et alii (2002), de ce personnage, c'est à dire le personnage qui fait avancer l'action et sur lequel les regards sont invités à se concentrer.

17. De fait, dans cette photo qui figure deux partners hommes en train de jouer au jeu de Go, une femme (directrice administrative du cabinet) est présente, entre les deux joueurs, et sa posture penchée, dans une attitude de prise de parole, semble indiquer qu'elle conseille un des joueurs. Toutefois, ce dernier semble également en train de parler.

18. Source interne CS2 and Partners (2012) : 33,9\%

\section{RÉSUMÉS}

L'article revient sur la place importante occupée par l'image, dans la communication institutionnelle et personnelle réalisée par les professionnel-le-s du conseil en management. A partir de deux groupes de ressources iconographiques, celles d'un bureau d'organisateurs conseils des années 1950 et celles d'un cabinet de conseil en management contemporain sont analysés les enjeux importants et la fonction de mise en récit que revêt l'image, dès l'émergence de ce groupe professionnel. Constituant une ressource pour les firmes en termes de construction d'une façade institutionnelle convaincante, elle participe en outre à la production de normes corporelles et esthétiques, adressant aux salarié-e-s des injonctions non neutres sur le plan du genre.

This article deals with the importance of iconography, in the institutional and personal communication of management consultants. Based on two groups of iconographic resources (one of a French pioneer firm of the 50's and one of a contemporary firm), the study analyzes the importance and the narrative functions of images, beginning with the early stages of the development of the sector. In this professional area, images turn out to be helpful resources to build legitimate "institutional façades" as well as to produce specific professional norms regarding body and appearance. Imposing specific social constraints for both male and female 
consultants, these norms are not gender neutral and participate to the resistance of gender barriers and inequality.

\section{INDEX}

Mots-clés : conseil en management, production visuelle, travail dramaturgique, façades institutionnelles, normes corporelles, genre

Keywords : management consulting, visual production, acting work, body norms, gender

\section{AUTEUR}

\section{ISABEL BONI-LE GOFF}

Sociologue du travail et du genre, Isabel Boni-Le Goff conduit des recherches sur les professions supérieures et leur féminisation. En prenant pour terrains les métiers techniques et le management, historiquement construits au masculin, ses travaux analysent les dynamiques qui traversent ces espaces professionnels, où la remise en cause des frontières de genre s'accompagne de multiples formes de résistance et de discrimination dans l'emploi, la carrière et dans l'accès aux fonctions dirigeantes. Dans une perspective compréhensive, se situant à la fois à l'échelle méso et microsociologique, elle croise approche quantitative et qualitative; elle réserve une place importante à l'ethnographie des situations de travail et à l'analyse comparée des productions indigènes qu'elles soient visuelles ou textuelles.

Docteure de l'EHESS, elle a obtenu un prix de l'Institut du Genre pour sa thèse"Le sexe de l'expert. Régimes de genre et dynamique des inégalités dans l'espace du conseil en management", qui a donné lieu à plusieurs publications. Depuis 2015, elle est chercheure à l'Université de Lausanne. 